\title{
Sobrepeso y obesidad en niños: implicaciones para la salud oral
}

\section{Overweight and obesity in children: oral health implications}

Pushparaja Shetty ${ }^{* *}$ y Akshatha Shetty ${ }^{2}$

${ }^{1}$ Departamento de Patología Oral; ${ }^{2}$ Departamento de Periodoncia. AB Shetty Memorial Institute of Dental Sciences, Nitte University, Deralakatte, Mangalore, India

Hemos leído el estudio original titulado "Prevalencia de sobrepeso y obesidad en niños escolares", de Emanuel de J. Torres-González et al., publicado en Gaceta Médica de México año 2020, volumen 156, páginas 182-186, ${ }^{1}$ y nos gustaría agradecer sus contribuciones. Es motivo de gran preocupación que el sobrepeso y la obesidad en los niños sea un problema global y se encuentre en tendencia creciente.

Los niños con sobrepeso y obesidad tienen más probabilidades de desarrollar numerosas enfermedades no transmisibles, como se menciona en el artículo, pero también tienen serias implicaciones en la salud oral. La obesidad y la salud oral están correlacionadas ya que comparten varios factores de riesgo comunes, incluidos los trastornos ambientales, genéticos, socioeconómicos y de estilo de vida. ${ }^{2}$ Los niños generalmente consumen grandes cantidades de bebidas azucaradas, bocadillos y alimentos ricos en azúcar que pueden causar caries localmente y obesidad en general. ${ }^{3}$ Numerosos investigadores están debatiendo la relación entre la obesidad y la caries dental, la enfermedad periodontal y la xerostomía. ${ }^{4}$

Históricamente se sabe que la caries dental y la enfermedad periodontal son uno de los desafíos más importantes en la salud pública, debido a sus efectos perjudiciales en el crecimiento y el desarrollo de los niños. ${ }^{5}$ El periodo de la infancia a la adolescencia es una etapa crítica de la vida en la que los niños desarrollan importantes hábitos de comportamiento relevantes para su salud general y oral. ${ }^{6}$

La Organización Mundial de la Salud destaca la necesidad de fortalecer las asociaciones existentes, la colaboración intersectorial, las sociedades comunitarias y las reformas de salud oral. ${ }^{3}$ Por lo tanto, necesitamos una estrategia global para prevenir la obesidad y sus consecuencias.

\section{Bibliografía}

1. Torres-González EJ, Zamarripa-Jáuregui RG, Carrillo-Martínez JM, Guerrero-Romero F, Martínez-Aguilar G. Prevalencia de sobrepeso y obesidad en niños escolares. Gac Med Mex. 2020;156:182-186.

2. Raghabendra P, Sowmya B, Jose $T$. The association between early childhood caries, body mass index and genetic sensitivity levels to the bitter taste of propylethiouracil (PROP) among the children of age 36-71 months. Int J Dent Update. 2012;2(1):24-32.

3. Petersen PE. The World Oral Health Report 2003: continuous improvement of oral health in the $21^{\text {st }}$ century-the approach of the WHO Global Oral Health Programme. Ginebra, Suiza: WHO; 2003.

5. Roa I, Del Sol M. Obesity, salivary glands and oral pathology. Colomb Med (Cali). 2018;49(4):280-287.

5. Cinar AB, Murtomaa $\mathrm{H}$. Interrelation between obesity, oral health and life-style factors among Turkish school children. Clin Oral Invest. 2011;15(2):177-184

6. Iglesia I, Doets EL, Bel-Serrat S, Román B, Hermoso M, Peña Quintana L, García-Luzardo MR, Santana-Salguero B, García-Santos Y, Vucic $\mathrm{V}$, et al. Physiological and public health basis for assessing micronutrient requirements in children and adolescents. The EURRECA network. Matern Child Nutr 2010;6 (Suppl 2):84-99. 